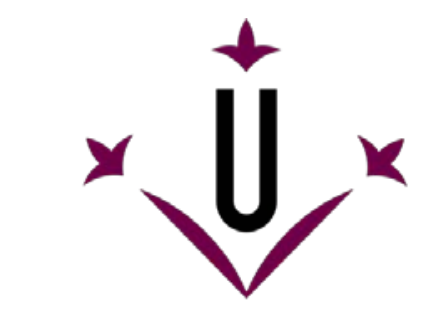

Universitat de Lleida

Document downloaded from:

http://hdl.handle.net/10459.1/72889

The final publication is available at:

https://doi.org/10.1109/JPHOTOV.2019.2937226

Copyright

(c) IEEE, 2019 


\title{
Fine-tuning of Multi-Junction solar cells: an in-depth evaluation
}

\author{
Laurène Parent, Alberto Riverola, Daniel Chemisana, Alain Dollet, and Alexis Vossier
}

\begin{abstract}
Multi-junction (MJ) solar cells are currently seen as the most promising technology toward achieving incomparable solar to electricity conversion efficiency, largely surpassing the best conventional single-junction solar cells. It was recently shown that futuristic cell architectures encompassing increasing number of subcells will likely show increased sensitivity to the spectral distribution of sunlight, giving rise to dramatic variations in the energy output of MJ-based PV systems from one location to the other. Here we investigate the extent to which MJ cells fine-tuned to the spectral-distribution of any particular site are likely to outperform conventionally-designed solar cells (tailored to AM1.5 reference solar spectrum). We first tackle the question of the extent to which fine-tuning may improve the performance of $\mathrm{MJ}$ cells showing deviations in the values of the main atmospheric parameters, relative to the reference solar spectrum. We then evaluate the potential of fine-tuning in improving the energy output of MJ cell-based PV systems for several locations selected for being representative of the broad diversity of atmospheric and climatic conditions. We demonstrate that the improvement in the energy output one can expect from fine-tuned PV cells strongly depends on how the mean annual spectrum deviates from the reference $A M 1.5$ solar spectrum, with values ranging from $\approx 0$ to more than $30 \%$, depending on the location and the cell architecture considered. Implications for future generations of multi-junction solar cells are finally discussed.
\end{abstract}

Index Terms-Multi-junction, photovoltaic cell, efficiency, sensitivity.

\section{INTRODUCTION}

$\mathbf{M}$ ULTI-junction solar cells are undoubtedly perceived as very promising candidates in the quest of ultraefficient PV cells. Today's solar to PV electricity efficiency record is held by a quadruple-junction solar cell with a conversion efficiency of $46 \%$ under concentrated sunlight [1]. Increasing the number of $p n$ junctions with appropriate band gaps leads to a more efficient utilization of the solar spectrum and consequently higher conversion efficiencies. Numerous technological and scientific challenges associated with the growth of multiple $p n$ junctions on top of each other [1] [2], or with the implementation of tunnel diodes between subcells, have been successfully overcome in the past years on increasingly complex cell architectures. 5 and 6 junction solar cells are currently investigated, with the tantalizing prospect of surpassing $50 \%$ solar to electricity conversion efficiency in the coming years [3]-[5]. Despite these promising perpectives, the development of efficient MJ solar cells involving a growing number of subcells will need further investigations,

Laurène Parent, Alain Dollet and Alexis Vossier are with CNRS, laboratoire PROMES, 7 Rue du Four Solaire, 66120 Odeillo, France

Alberto Riverola and Daniel Chemisana are with Universitat de Lleida, Lleida, Spain

Corresponding Author, e-mail : alexis.vossier@promes.cnrs.fr for example into the enhanced capacity of these cells to better absorb light. A particular feature of MJ cells, stemming from the electrical interconnection scheme commonly followed in the vast majority of today's MJ cells technologies, lies in their increased sensitivity to the spectral distribution of sunlight. The series connections between subcells imposes an upper limit to the current extractable from the MJ cell, which should be equal to the lowest current generated in each individual subcell. As a consequence, minor alterations in the spectral distribution of light associated with variations in the main atmospheric parameters, may have a significant impact on the ability of MJ cells to efficiently convert sunlight into electricity. The ability of MJ cells comprising a high number of subcells to accommodate changes in the spectral distribution of light was recently questioned [6]-[8]. In particular, the energy output of CPV systems was shown to be highly dependent on the climatic conditions to which they are exposed. As a consequence, using MJ cells involving increasing number of subcells does not necessarily guarantee a higher energy production over extended periods of time. These findings may constitute a paradigm shift in the way advanced MJ solar cells should be designed: rather than aiming at the highest solar to electricity conversion efficiency considering a unique reference solar spectrum (typically ASTM G173 AM1.5 solar spectrum [9]), advanced MJ solar cells should be tailored to ensure a higher energy output over extended periods of time. This question was recently investigated by several authors [10]-[12]). Yandt et al considered representative spectrum, using AM as a proxy for describing spectral changes, to tailor MJ cells comprising up to 10 subcells [10]. They showed a significant improvement in the energy output achievable, especially for PV cells involving a high number of subcells and for high-latitude locations. Garcia et al suggested a binning algorithm aiming to generate "proxy" spectra accounting for the spectral variability at a specific location [12]. This work aims at shedding light on the ability of fine-tuning to better harness solar energy, considering the most significant atmospheric parameters. Using a visual representation, we first try to grasp which atmospheric parameters are likely to require a fine-tuning of the cell architecture for maximizing the energy produced. Then, we evaluate the amplitude with which the spectral distribution of sunlight may deviate from the reference $A M 1.5$ solar spectrum, considering 5 locations chosen for being representative of the wide variety of climatic conditions suitable for PV. Finally, the last section of this article is devoted to a systematic study aiming to assess the benefit of fine-tuning by comparing the annual yield of CPV systems involving 1) fine-tuned and 2) conventional MJ solar cells (i.e. cells designed for an optimal conversion of $A M 1.5$ 
solar spectrum).

\section{METHODOLOGY}

\section{A. Bandgap optimization}

Multi-junction solar cells are commonly designed assuming a spectral distribution of the incoming light described by the AM1.5 reference solar spectrum (ASTM G173). Procedures aiming to determine the optimal combination of bandgaps leading to the highest conversion efficiency have already been described by other authors ( [13]-[15]). In this work, we aim to tailor MJ cell architectures to the mean spectral content of the light absorbed over an extended period of time. To this end, we developed a genetic-algorithm based method able to define the optimal set of bandgap combination leading to the highest solar to electricity conversion efficiency, using as an input the spectral distribution of light. Genetic algorithms are optimization tools basically inspired by natural selection, used to solve complex optimization problems involving several parameters [16]. This method offers a rapid and powerful alternative to other optimization tools, with reduced computational time and high accuracy (with typical calculation times of $\sim 60$ minutes instead of $\sim 1.5$ days for more standard methods, such as the one described in [7]). It is based upon the minimization of an objective function (defined here as 1- $\eta$ where $\eta$ refers to the conversion efficiency of the MJ stack investigated): applying successively several genetic operators, the genetic algorithm searches for a set of parameters leading to a minimum value of the objective function. In this particular problem, the search space, inside which the optimal set of parameters is scrutinized, consists of the bandgaps of the semiconductor materials involved in the stack (their number being dependent upon the cell architecture investigated). From an initial set of individuals, randomly selected, a new population is generated using various genetic operations, namely:

1) Selection: this operation selects the best individuals based on their fitness value, and transmits their traits to the next generation. It allows more copies of the best individuals with higher scores, hence giving a larger survival probability to these individuals. If only selection is used, the new generation is an exact copy of the best parents.

2) Cross - over: this operation combines traits from two selected parents to form a new solution. The traits are randomly exchanged between the parents, generating new individuals that are completely different from the previous ones. The parents' traits with higher scores are favored in the mix, to make the population evolve toward the best possible solution.

3) Mutation: this operation applies random changes to the parents' solution to create new sets of individuals, to maintain diversity among the population.

Alternative methods described elsewhere ( [7] [13] [14] [15]) could have been used for performing the optimization. The accuracy of the proposed method was evaluated as follows: the optimal set of bandgaps was computed assuming an AM1.5 spectral distribution of the incident light, and compared with previously published data obtained from an alternative methods ( [7]). The excellent agreement between these 2 methods, with discrepancies in the optimum predicted $E_{g}$ values of no more than $0.01 \mathrm{eV}$, stresses the ability of genetic algorithms to accurately determine the optimal set of bandgaps for MJ cell architectures comprising up to 10 subcells.

\section{RESULTS}

\section{A. Influence of the atmospheric parameters}

It was previously shown that MJ solar cells comprising increasing number of subcells are likely to show lower performance under illumination conditions deviating from the reference conditions ( [8] [17]). These previous findings raise the question of how cells tailored to better match the spectral distribution of sunlight at any particular location are likely to outperform conventional MJ cells. This can be assessed by comparing the conversion efficiency achievable with both types of cells exposed to sunlight whose spectral distribution deviates from the $A M 1.5$ reference spectrum. Fig. 1 shows the relative improvement in the fine-tuned MJ cells' ability to convert sunlight into electricity, as a function of the $A M$, $A O D_{500}$ and $P W$ values, and for MJ stacks comprising up to 10 subcells (the reference value for each atmospheric parameter being indicated by a white dotted line). Blueish colors indicate a modest improvement in the cell efficiency relative to $A M 1.5$-optimized $\mathrm{MJ}$ cells, while the reddish areas correspond to the most significant efficiency increase.

In the light of these results, one can reach 2 important preliminary conclusions: 1) the extent to which fine-tuning of PV cells may lead to higher performance depends upon the atmospheric parameter which deviates from the reference conditions: if exposed to sunlight characterized by noticeable variations in $A M$ or $A O D_{500}$, a fine-tuned $\mathrm{PV}$ cell may show significantly higher conversion efficiency relative to conventional PV cells. Conversely, there is almost no benefit for fine-tuning PV cells exposed to light whose spectral distribution is only affected by PW values deviating from their reference values; 2) the benefit for fine-tuning is highly dependent on the cell architecture considered: while singlejunction cells appear to be practically insensitive to variations in the main atmospheric parameter values, we observe a significant improvement in the efficiency of fine-tuned MJ cells involving a high number of subcells.

In reality, the spectral distribution of the light impinging the cell is a complex function of the 3 atmospheric parameters, which are likely to vary simultaneously. A precise assessment of fine-tuning should thus take into account the combined effects of $A M, A O D_{500}$ and $P W$, as illustrated in Fig. 2.

The 3 upper graphs show the gain in efficiency (relative to $A M 1.5)$ achievable with cells exposed to simultaneous deviations in both $A M$ and $A O D_{500}$ values, for singlejunction cells (Fig. 2 a1), 5-junction cells (Fig. 2 a2) and 10 junctions cells (Fig. 2 a3). The intermediate and lower graphs depict the relative gain in efficiency achievable with fine-tuned PV cells, considering simultaneous variations of $A M$ and $A O D_{500}$ (Fig. $2 b 1, b 2$, and $b 3$ ), and $A O D_{500}$ and $P W$ (Fig. $2 c 1, c 2$, and $c 3$ ). In each case, one atmospheric 


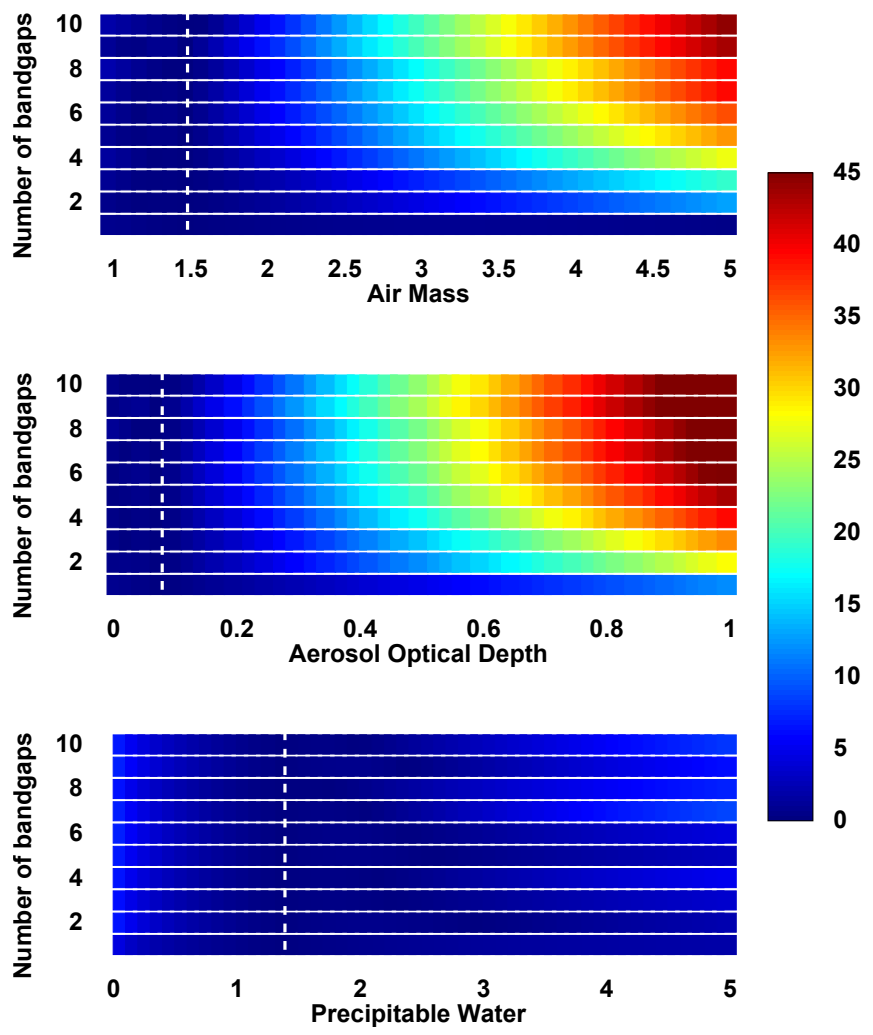

Fig. 1. Variation in the efficiency achievable with a fine-tuned solar cell as a function of the atmospheric parameter values, relative to AM1.5D-optimized solar cells, and assuming an illumination of 1000 suns. The white dotted line indicates the value associated with the AM1.5 reference spectrum

parameter $\left(P W\right.$ in the upper graphs, $A O D_{500}$ in the middle graphs, and $A M$ in the lower graphs) is set constant and equal to the reference $A M 1.5 D$ ( $A S T M G 173$ ) value. For improved clarity, the white crosses indicate the combination of atmospheric parameters associated to the $A M 1.5 D$ reference values (the corresponding efficiencies are indicated into brackets, to provide a reference point for the reader). In addition, we also report the mean atmospheric parameter values (averaged over one year) for 5 locations selected for being representative of the broad range of atmospheric and climatic conditions (see table I for the corresponding numerical values). For a particular cell architecture, the benefit for using fine-tuned solar cells can be deduced from the typical color associated with a given set of atmospheric parameters. As for fig. 2, the efficiency gain is associated with a color scale (dark blue = no gain, dark red $=$ maximum gain). Fig. 2 reveals some important conclusions regarding this strategy: 1) The benefit for finetuning is, as already suggested in Fig. 1, largely dependent upon the number of subcells involved in the MJ stack. The range of atmospheric parameters values for which the cell efficiency peaks tends to narrow significantly with increasing number of subcells. In particular, there is a noticeable benefit for fine-tuning MJ solar cells involving 5 or 10 subcells, as soon as the typical atmospheric parameters deviate slightly from the $A M 1.5$ reference values. 2) Combined variations of two different atmospheric parameters do not affect the spectral distribution of light (and consequently the benefit for finetuning) in comparable fashions. We notice a more pronounced tolerance of $\mathrm{PV}$ cells to combined variations in $P W$ and $A M$, or $P W$ and $A O D_{500}$, than to combined variations in $A M$ and $A O D$. This translates into a higher benefit for fine-tuning PV cells likely to face deviations in both the $A M$ and $A O D_{500}$ values: for instance, both Xianghe and Nauru demonstrate significant deviations in their mean atmospheric parameter values, relative to the AM1.5 reference values. In the case of Xianghe, these variations are particularly noticeable in the AOD Vs AM (Fig. $2 a 1, a 2$, and $a 3$ ) and AOD Vs PW (Fig. $2 c 1, c 2$, and $c 3$ ) graphs. Conversely, the deviations in the case of Nauru are essentially discernible in the PW Vs AM (Fig. 2 b1, b2, and b3) as well as PW Vs AOD (Fig. $2 c 1$, $c 2$, and $c 3$ ) graphs, whose combined variation are less likely to affect the spectral distribution of light. As a consequence, even if these two cities show significantly different mean atmospheric parameter values (comparatively to the AM1.5 reference values), the motivation for fine-tuning PV cells is expected to be higher for the former city.

\section{B. Spectral simulation}

The benefits for fine-tuning MJ solar cells to the spectral distribution of the incoming light is a complex function of the main atmospheric parameters. Therefore we perform a systematic study aiming to evaluate the gain in the energy output of CPV systems involving fine-tuned MJ solar cells. Five different locations, fulfilling several fundamental data quality requirements [8], are selected: the mean solar spectrum, averaged over a full typical working year, is first calculated (a detailed description of the spectrum calculation, based on the use of AERONET data [18] and SMARTS software can be found in [8]). The selected cities cover a wide range of latitudes (see details in table I), extending from Nauru (Micronesia) to Carpentras (southern France). Xianghe (China) is characterized by a high pollution level giving rise to large $A O D_{500}$ values. Nauru is a tropical site with high $P W$, while Sede Boqer (Israel) and Casleo (Argentina) are representative of arid climatic conditions. The mean annual values of the main atmospheric variables and the site altitude, also reported in table I, allow the calculation of the mean spectral distribution of sunlight over a year. Fig. 3 depicts the mean annual spectrum for each investigated location (red curve), together with the $A M 1.5$ solar spectrum (blue curve) and the deviation relative to $A M 1.5$ spectrum (dashed black curve). The spectra are normalized so that the total solar irradiance corresponding to each spectrum equals the $A M 1.5 D$ solar spectrum power density $\left(900 \mathrm{~W} / \mathrm{m}^{2}\right)$. Several conclusions can be drawn from this figure:

Changes in the mean annual atmospheric parameters may significantly alter the spectral distribution of light: we observe a strong imbalance in the UV/Vis/IR distribution relative to $A M 1.5$ spectrum for locations such as Xianghe and Carpentras, which are respectively characterized by high mean $A O D_{500}$ (Xianghe) and high mean $A M$ (Carpentras and Xianghe).

The potential interest for fine-tuning can be inferred from these preliminary observations: locations showing significant 

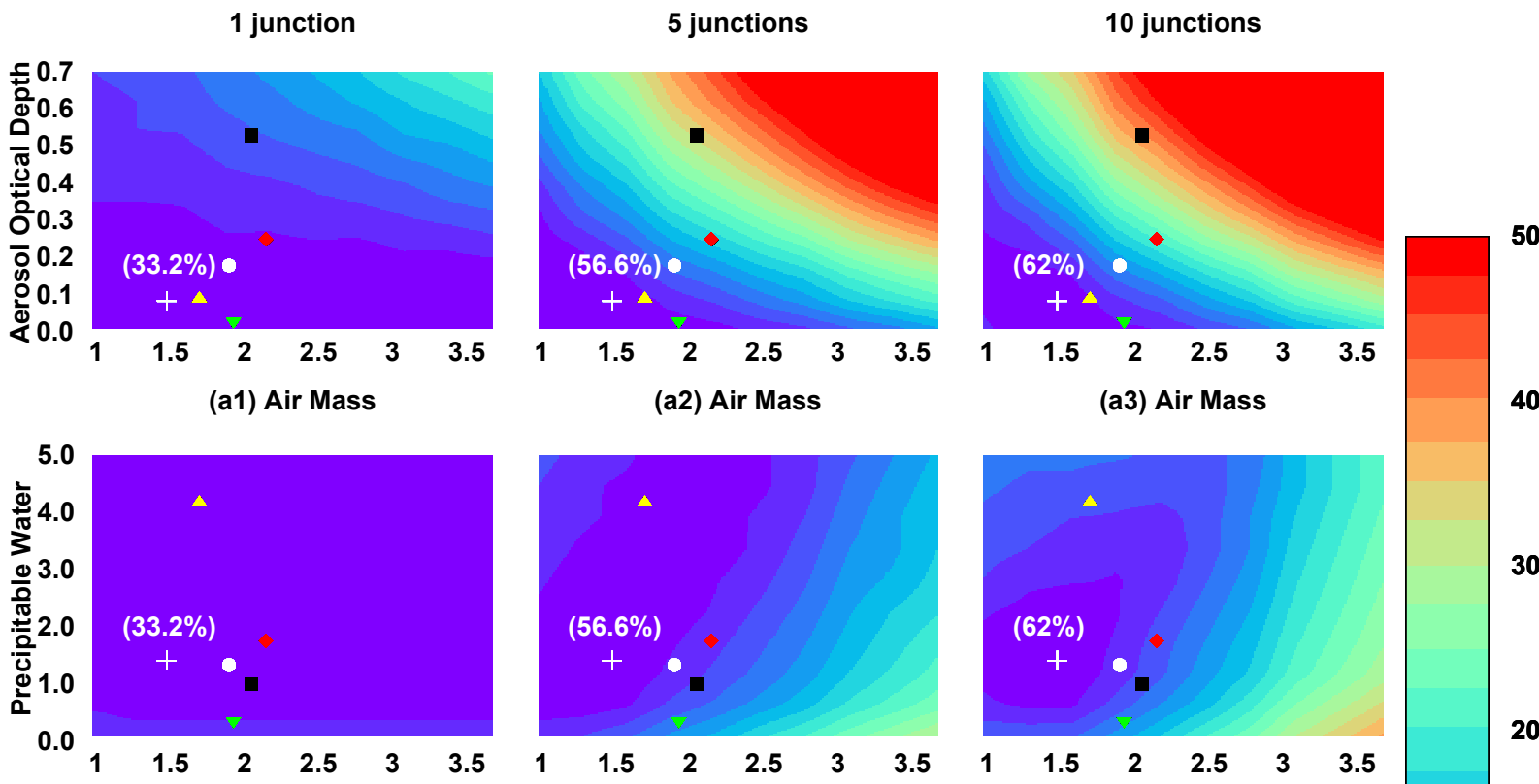

(b1) Air Mass

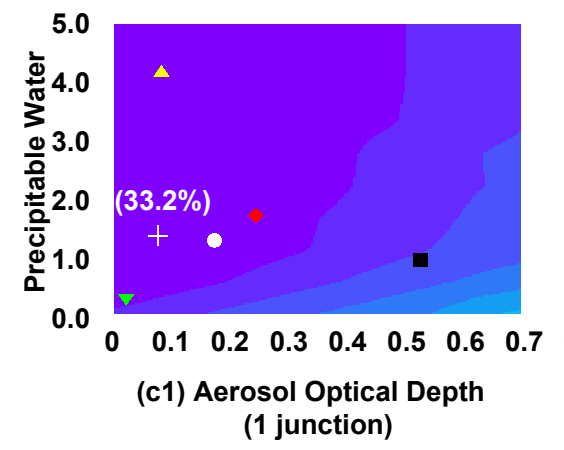

(b2) Air Mass

(b3) Air Mass
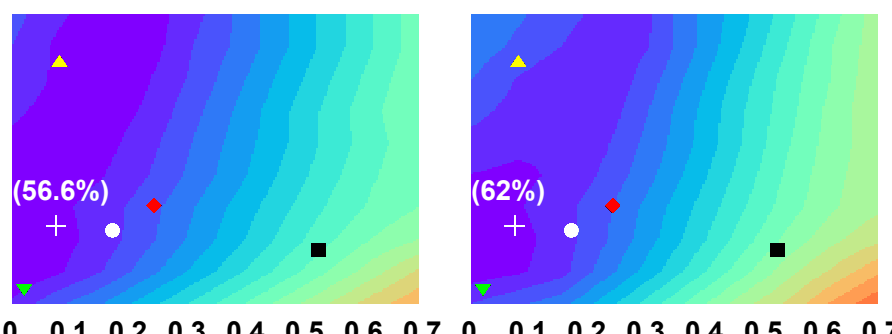

(c2) Aerosol Optical Depth (5 junctions)

(c3) Aerosol Optical Depth (10 junctions)

Fig. 2. Relative variation (\%) in the efficiency achievable with a fine-tuned solar cell as a function of the atmospheric parameter value. The white crosses indicate the combination of atmospheric parameters corresponding to the $A M 1.5$ reference values. The colored symbols represent the combination of the mean annual atmospheric parameters values for several representative locations around the world ( $\triangle$ : Nauru (Micronesia) - $\bigcirc$ : Sede Boker (Israel) - a: Xianghe (China) - $\bullet$ : Carpentras (France)- $\nabla$ : Casleo (Argentina))

deviations in the mean spectral distribution of light are expected to demonstrate higher benefit from fine-tuning. The noticeable red-shift in the mean spectral distribution observed in Xianghe and Carpentras may lead to fined-tuned MJ cells characterized by lower bandgap values relative to conventional AM1.5-designed solar cells.

The optimal combination of bandgaps is calculated with the genetic algorithm optimization method for cell architectures involving up to 10 subcells, and using as an input the mean annual spectrum. The energy output of CPV modules is then calculated for both conventional and fine-tuned MJ cells, following the procedure described in [6] [8], over a full year's operation (the optimal combinations of bandgap for each city investigated are reported in the supplementary material). Fig. 4 shows the relative improvement in the energy output of a CPV module based on conventional (blue bars) and fine-tuned solar cells (green bars), assuming a reference output energy value corresponding to conventional double- junction cells (Sede Boker is voluntarily omitted among the locations represented, because of the minor difference in the energy output between AM1.5 - optimized and fine-tuned cells). One can distinguish different trends depending on the location and the typical atmospheric conditions considered: locations characterized by an annual spectral distribution of light close to $A M 1.5$ spectrum, such as Sede Boker or Casleo, do not show any significant benefit from fine-tuning (the improvement in the energy output does not exceed $1 \%$, even for cell architectures involving up to 10 subcells). We can draw different conclusions for locations showing less favorable atmospheric parameters, namely: 1) reduced ability of MJ cell architectures involving a high number of subcells to provide a boost in the energy output equivalent to the one obtained in more favorable locations; 2) improved benefit for fine-tuning MJ cells comprising a high number of subcells (which can be deduced from the noticeable gain in the efficiency output achievable using fine-tuned cells relative to conventional 
TABLE I

ANNUAL MEAN ATMOSPHERIC PARAMETER VALUES FOR THE DIFFERENT SELECTED LOCATIONS

\begin{tabular}{c|c|ccc|c|ccc}
\hline \hline & & \multicolumn{4}{|c|}{ Spatial coordinates } & \multicolumn{3}{|c}{ Annual mean atmospheric parameters } \\
\hline Location & Simulated year & $\begin{array}{c}\text { Lat } \\
\left({ }^{\circ}\right)\end{array}$ & $\begin{array}{c}\text { Long } \\
\left({ }^{\circ}\right)\end{array}$ & $\begin{array}{c}\text { Elevation } \\
(\mathrm{m})\end{array}$ & $\begin{array}{c}\text { Mean Daily DNI } \\
\left(k W h / \mathrm{m}^{2} / \text { day }\right)\end{array}$ & $A M$ & $\begin{array}{c}P W \\
(\mathrm{~cm})\end{array}$ \\
\hline$A M 1.5$ & & & & & & 1.5 & 0.084 \\
\hline Nauru $(\triangle)$ & 2011 & -0.52 & 166.92 & 7 & $6-6.5$ & $1.72(+10 \%) *$ & $0.09(+10 \%) *$ & $4.17(+200 \%) *$ \\
Sede Boker $(\circ)$ & 2004 & 30.86 & 34.78 & 180 & $6.5-7$ & $1.92(+30 \%) *$ & $0.18(+110 \%) *$ & $1.34(-10 \%) *$ \\
Xianghe $(\square)$ & 2009 & 39.75 & 116.96 & 32 & $5.5-6$ & $2.07(+40 \%) *$ & $0.53(+530 \%) *$ & $1.01(-30 \%) *$ \\
Carpentras $(\diamond)$ & 2003 & 43.93 & 4.88 & 32 & $5-5.5$ & $2.17(+40 \%) *$ & $0.25(+200 \%) *$ & $1.76(+20 \%) *$ \\
Casleo $(\nabla)$ & 2011 & 31.80 & 69.31 & 2552 & $6.5-7$ & $1.93(+30 \%) *$ & $0.03(-60 \%) *$ & $0.37(-70 \%) *$ \\
\hline \hline
\end{tabular}

${ }^{*}$ In brackets: local atmospheric parameter variation relative to AM1.5 reference conditions
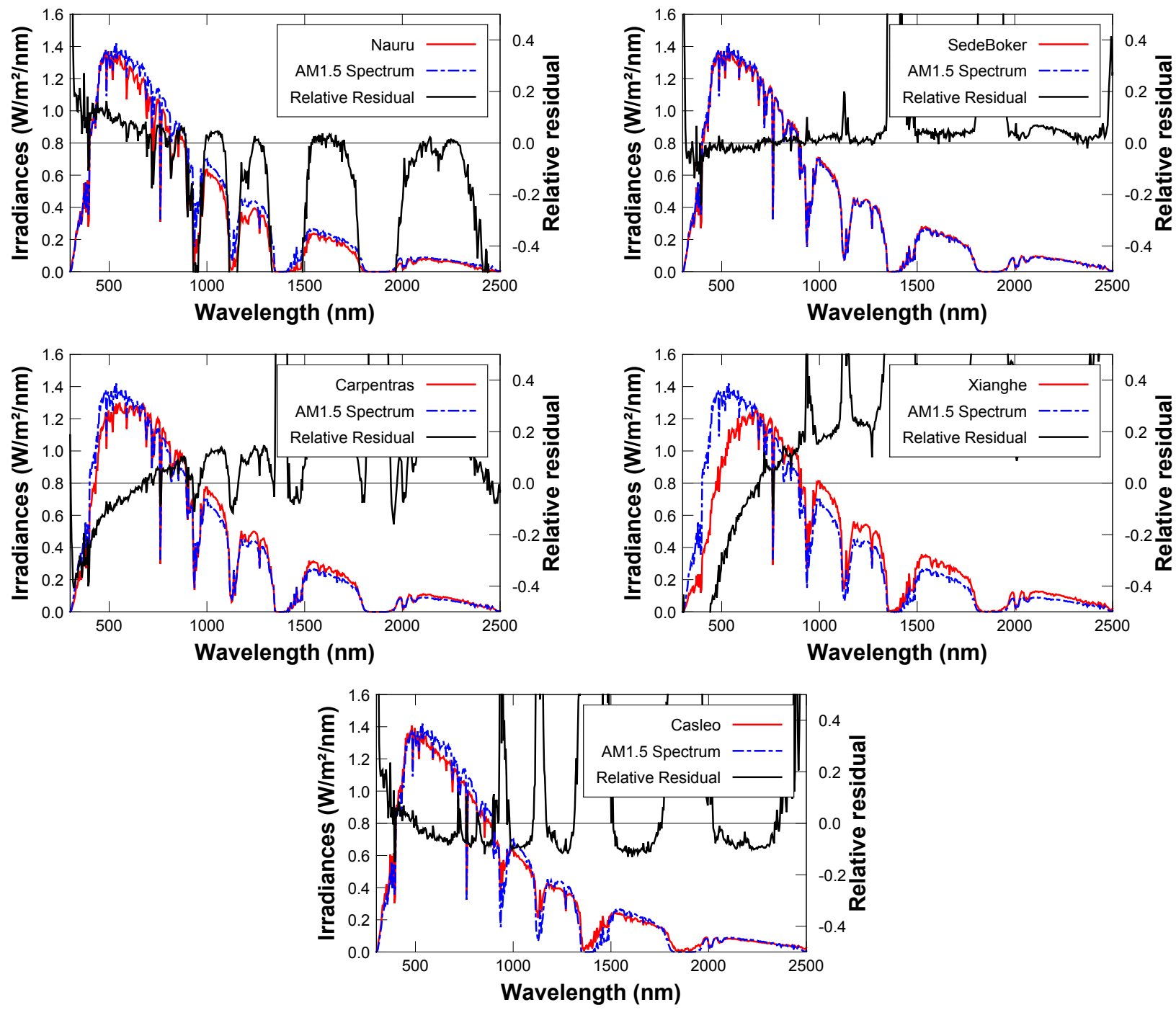

Fig. 3. Mean annual solar spectrum -normalized to a power density of $900 \mathrm{~W} / \mathrm{m}$ (red), AM1.5 spectrum (blue) and relative difference between the 2 spectra (black)

\section{AM1.5-designed cells).}

Finally, there is a dramatic improvement in the energy output of CPV systems involving fine-tuned cells in locations characterized by a mean annual spectrum deviating significantly from the $A M 1.5$ reference spectrum In the case of Xianghe, the relative energy output appears to be lower than 1 for AM1.5D-optimized MJ cells comprising 5 subcells and more: this illustrates the deficiency of $A M 1.5$ solar spectrum to describe the mean spectral distribution in this particular place (principally because of the high $A O D$ mean value). In this case, fine-tuning the bandgap combination allows the energy output to be drastically improved. The gain in the relative energy output approaches 1.3 in the case of MJ cells 
a) Nauru

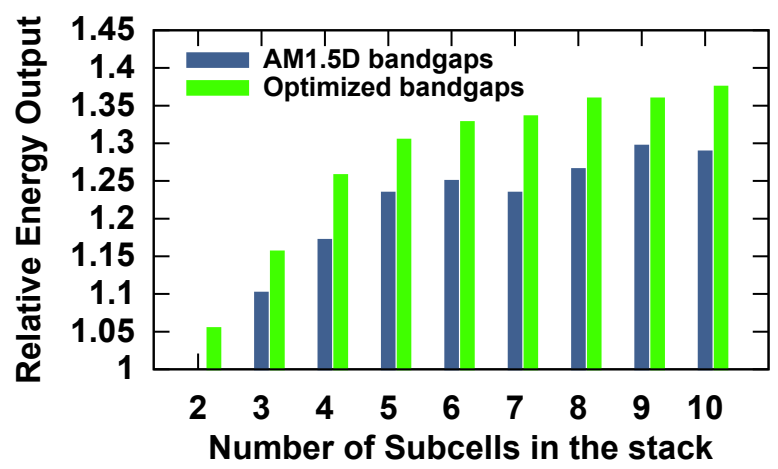

c) Carpentras

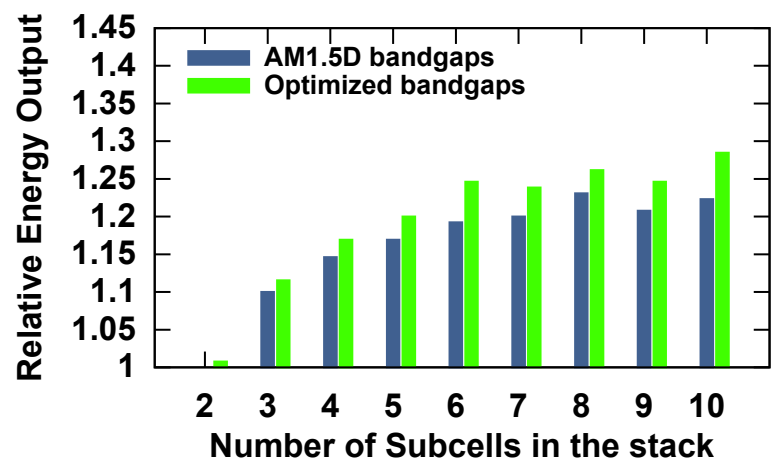

b) Xianghe

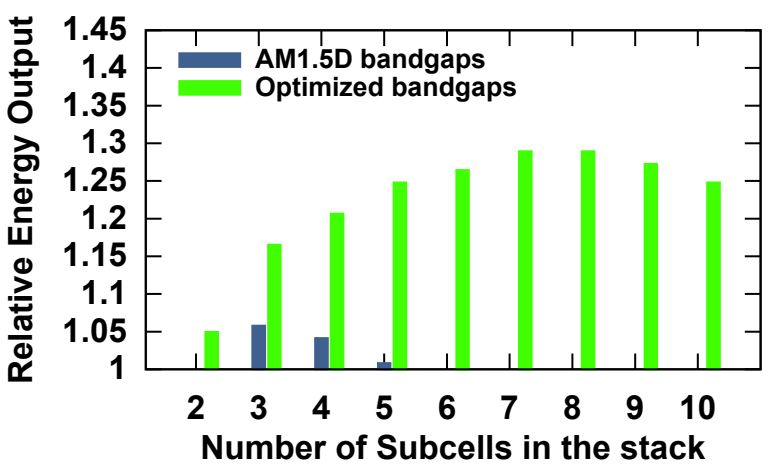

d) Casleo

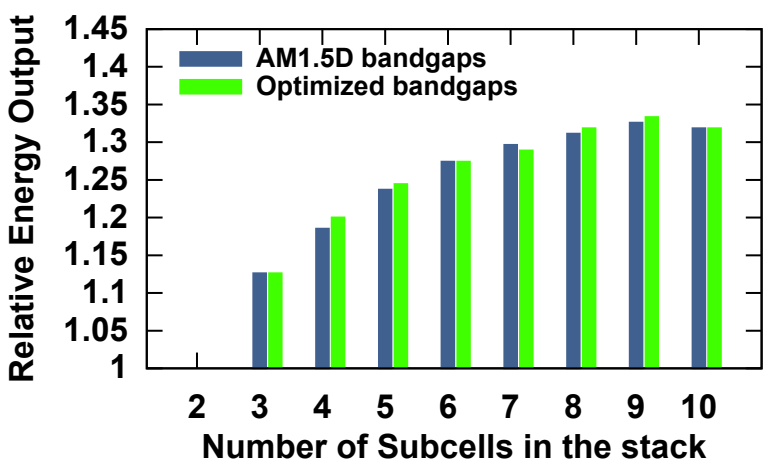

Fig. 4. Relative variation in the efficiency achievable with fine-tuned solar cells for the selected locations, and for cell architectures comprising between 2 and 10 subcells

comprising 7 or 8 subcells, which is comparable to the value observed in more favorable locations.

\section{CONCLUSION}

The question of the most efficient design allowing MJ cells to better harness solar energy becomes more intricate as the architecture of MJ solar cells becomes more complex. The increased sensitivity to the spectral distribution of sunlight associated with a high number of subcells was previously shown to significantly lower the energy output of MJ-based CPV systems. Here, we provided an in-depth evaluation of fine-tuning, i.e. the strategy by which a PV cell is tailored to the mean spectral distribution of a given location, rather than to a reference solar spectrum.

The benefit for fine-tuning was shown to be highly dependent on: 1) the nature of the atmospheric parameters likely to vary; 2) the cell architecture (in particular the number of subcells involved in the MJ stack). While conventional PV cells demonstrate a good ability to accomodate variations in $P W$, we observed a strong benefit for fine-tuning PV cells exposed to varying $A M$ and $A O D_{500}$ values. The gain in efficiency provided by fine-tuning depends largely upon the number of $p n$ junctions involved in the MJ stack, as well as the extent to which both parameters vary relative to the reference $A M 1.5$ value.
The improvement in the energy output one can expect from fine-tuned PV cells strongly depends on how the mean annual spectrum deviates from the reference $A M 1.5$ solar spectrum: locations whose mean-annual spectrum is accurately described by $A M 1.5$ solar spectrum do not show any significant improvement in the use of fine-tuned PV cells. On the other hand, there is an increasing incentive to adapt the cell architecture if the mean annual spectrum noticeably deviates from $A M 1.5$ solar spectrum. Indeed, the gain in the maximum energy output achievable is particularly noticeable for cell architectures involving a high number of $p n$ junction. Due to the high operational costs associated with their design and fabrication, the implementation of fine-tuned cells may only be feasible in places with a mean annual spectral distribution deviating significantly from $A M 1.5$ reference spectrum (the standard bandgap design calculation methods being effective for places showing low to moderate deviations in their mean spectral distribution). However, CPV systems should obviously be designed to ensure the highest energy yield possible, a condition which is far from being fulfilled with conventional MJ cells encompassing a high number of subcells. These results suggest that alternative design strategies should be further investigated to this aim. 


\section{REFERENCES}

[1] F. Dimroth, T. N. Tibbits, M. Niemeyer, F. Predan, P. Beutel, C. Karcher, E. Oliva, G. Siefer, D. Lackner, P. Fuß-Kailuweit et al., "Four-junction wafer-bonded concentrator solar cells," IEEE Journal of Photovoltaics, vol. 6, no. 1, pp. 343-349, 2016.

[2] F. Dimroth, M. Grave, P. Beutel, U. Fiedeler, C. Karcher, T. N. Tibbits, E. Oliva, G. Siefer, M. Schachtner, A. Wekkeli et al., "Wafer bonded four-junction gainp/gaas//gainasp/gainas concentrator solar cells with 44.7\% efficiency," Progress in Photovoltaics: Research and Applications, vol. 22, no. 3, pp. 277-282, 2014.

[3] P. Chiu, D. Law, R. Woo, S. Singer, D. Bhusari, W. Hong, A. Zakaria, J. Boisvert, S. Mesropian, R. King et al., "Direct semiconductor bonded $5 \mathrm{j}$ cell for space and terrestrial applications," IEEE Journal of Photovoltaics, vol. 4, no. 1, pp. 493-497, 2014.

[4] P. Chiu, D. Law, S. Singer, D. Bhusari, A. Zakaria, X. Liu, S. Mesropian, and N. Karam, "High performance $5 \mathrm{j}$ and $6 \mathrm{j}$ direct bonded (sbt) space solar cells," in Photovoltaic Specialist Conference (PVSC), 2015 IEEE 42nd. IEEE, 2015, pp. 1-3.

[5] J. F. Geisz, M. A. Steiner, N. Jain, K. L. Schulte, R. M. France, W. E. McMahon, E. E. Perl, and D. J. Friedman, "Building a sixjunction inverted metamorphic concentrator solar cell," IEEE Journal of Photovoltaics, vol. 8, no. 2, pp. 626-632, 2018.
[6] N. L. A. Chan, H. E. Brindley, and N. J. Ekins-Daukes, "Impact of individual atmospheric parameters on cpv system power, energy yield and cost of energy," Progress in Photovoltaics: Research and Applications, vol. 22, no. 10, pp. 1080-1095, 2014.

[7] A. Vossier, E. Al Alam, A. Dollet, and M. Amara, "Assessing the efficiency of advanced multijunction solar cells in real working conditions: a theoretical analysis," IEEE Journal of Photovoltaics, vol. 5, no. 6, pp. 1805-1812, 2015.

[8] A. Vossier, A. Riverola, D. Chemisana, A. Dollet, and C. A. Gueymard, "Is conversion efficiency still relevant to qualify advanced multi-junction solar cells?" Progress in Photovoltaics: Research and Applications, vol. 25 , no. 3, pp. 242-254, 2017.

[9] A. Standard, "G173,standard tables for reference solar spectral irradiances: Direct normal and hemispherical on 37 tilted surface, amer," Society for Testing Matls., West Conshocken PA, USA, 2007.

[10] M. D. Yandt, K. Hinzer, and H. Schriemer, "Efficient multijunction solar cell design for maximum annual energy yield by representative spectrum selection," IEEE Journal of Photovoltaics, vol. 7, no. 2, pp. 695-701, 2017.

[11] E. C. Warmann and H. A. Atwater, "Predicting energy production for multijunction photovoltaics: Effects of spectral variation and cumulative irradiance," in Photovoltaic Specialists Conference (PVSC), 2016 IEEE 43rd. IEEE, 2016, pp. 0577-0579.

[12] I. Garcia, W. E. McMahon, A. Habte, J. F. Geisz, M. A. Steiner, M. Sengupta, and D. J. Friedman, "Spectral binning for energy production calculations and multijunction solar cell design," Progress in Photovoltaics: Research and Applications, vol. 26, no. 1, pp. 48-54, 2018.

[13] A. De Vos, "Detailed balance limit of the efficiency of tandem solar cells," Journal of Physics D: Applied Physics, vol. 13, no. 5, p. 839, 1980.

[14] A. Martí and G. L. Araújo, "Limiting efficiencies for photovoltaic energy conversion in multigap systems," Solar Energy Materials and Solar Cells, vol. 43, no. 2, pp. 203-222, 1996.

[15] A. S. Brown and M. A. Green, "Limiting efficiency for currentconstrained two-terminal tandem cell stacks," Progress in Photovoltaics: Research and Applications, vol. 10, no. 5, pp. 299-307, 2002.

[16] S. Sivanandam and S. Deepa, "Genetic algorithms," in Introduction to genetic algorithms. Springer, 2008, pp. 15-37.

[17] J. Villa and A. Martí, "Impact of the spectrum in the annual energy production of multijunction solar cells," IEEE Journal of Photovoltaics, vol. 7, no. 5, pp. 1479-1484, 2017.

[18] D. Knuth. Aerosol robotic network (aeronet) homepage. [Online]. Available: http://aeronet.gsfc.nasa.gov 